\title{
A Short Discussion of Network Analysis
}

\author{
Fengrong Wei* \\ Department of Mathematics, University of West Georgia, USA
}

Submission: March 15, 2018; Published: June 21, 2018

*Corresponding author: Fengrong Wei, Department of Mathematics, University of West Georgia, USA; Tel: 678-8395314; Email: fwei@westga.edu

\begin{abstract}
Network analysis has become a significant tool for understanding the dynamics of complex system. This paper provides a short overview of several network methods and their applications. In addition, a discussion of potential future works in network analysis is provided.

Keywords: Network; Multiple connections; Dynamic; Network analysis; Complex system; Neurological; Heterogenous connections; Diagonal of matrix; Bayesian nonparametric model; Dynamic binary relational matrix; Time series dynamic; Time-varying; Complexity; Hierarchical structure; Social network; Interconnected data; Computational methods; Theoretical properties; Symmetric
\end{abstract}

\section{Introduction}

Due to the rapid development of technology and the increasing complexity of many studied areas, network analysis becomes popular and necessary nowadays [1-5]. Subjects within a studied network could be connected with each other through various different types of relationships. For example, biological systems can be thought of a complex network, such that every biological entity interacts with other biological entities at different systemic levels, including ecological, neurological, metabolic, etc, resulting in heterogenous connections. Analyzing the effect of individual connections and the interaction of different connections helps understanding the mechanisms of the network system. During recent years, researchers have developed a variety of techniques and models to help understand or predict the effects of connections on a response variable.

Consider a study of $N$ subjects (e.g., biological entity in biological network), which are equivalent to the $\mathrm{N}$ nodes in the network study. These $\mathrm{N}$ nodes are connected with each other through $\mathrm{p}$ different relationships. Let an adjacency matrix $A^{k}=\left(a_{i, j}^{k}\right) \in \mathbb{R}^{N \times N}$ describe the $k^{\text {th }}$ relationship among the $N$ nodes, $k=1, \ldots, p$. If there exists the $k^{\text {th }}$ relation between nodes $i$ and $j$, then $a_{i, j}^{k}=1$, and $a_{i, j}^{k}=0$ otherwise. The diagonal of matrix $A^{k}$ is set to be zero, which does not allow any node to be self-related. Moreover, for each type of connection, the adjacency matrices does not need to be symmetric, which

could represent either a directed or an undirected connection. Durante \& Dunson [2] proposed a Bayesian nonparametric model and inferred the time-varying effects of $A^{k}$ on a dynamic binary relational matrix, which can be considered as a network type response variable. Zhu et al. [3] considered a large-scale network, i.e., one $A^{k}$, with a continuous response variable observed for each node at equally spaced time points, and studied the time series dynamic of the responses. Wei \& Tian [5] extended the study of Zhu et al. [3] from one network to multiple connections and studied the heterogeneous effects of various connections on the responses. Certainly, there are many other more studies in the network analysis and their applications.

In addition to these existing studies, there are many works worth to be studied in future. For example, although subjects within a studied network could be connected through various relationships $A^{k}$, it is possible that not all $A^{k}$ are important for a studied outcome and the effects of the important connections on the studied outcome could be quite different. Then it is interesting to investigate the selection of the important connections and the estimation of those selected important connections' effects on the response variable simultaneously. Moreover, the relationship $A^{k}$ can be time-varying, i.e., $A_{t}^{k}$. Then the selection and estimation of the dynamic effects of these heterogenous connections are also interesting. Another example, the complexity of a network system may not only come from the heterogenous types of connections, but also the hierarchical structure embedded in the network system. For instance, an internal connection exists within each node in the network system, i.e, for each node $i, j=1, \cdots, N$, there exists an internal network which can be described by an adjacency matrix $M^{i}$. Thus, the network system is a two-layer network, the first layer $\left(A^{k}\right)$ describes the connections among the $N$ subjects, such as patients' social network, and the second layer $\left(M^{i}\right)$ describes the connections within each subject, such as the gene connections within each patient. Then, this hierarchical network could be applied to investigate the disease related genes and the spill over effect of this disease across $N$ subjects. Taking 
the hierarchical structure into the study helps understanding the channel of contagion and the fundamental of the studied disease.

\section{Conclusion}

Due to the rapid development of new technology as well as the growth of society, subjects in a network become complexly connected with others through a variety of relationships. Network analysis becomes necessary and important, it helps understand the behavior of complex and interconnected data, providing insight in a world of complexity. This paper provides a short review of some network analysis methods and discusses several future works in network analysis. Both computational methods and theoretical properties are needed in future studies.

\section{References}

1. Durante D, Dunson D (2014) Nonparametric Bayes dynamic modelling of relational data. Biometrika 101(4): 883-898.

2. Durante D, Dunson D (2014) Bayesian dynamic financial networks with time-varying predictors. Statistics \& Probability Letters 93: 1926.

3. Zhu X, Pan R, Li G, Liu Y, Wang H (2017) Network vector autoregression The Annals of Statistics 45(3): 1096-1123.

4. Fujita A, Takahashi DY, Balardin JB, Vidal MC, Sato JR (2017) Correlation between graphs with an application to brain network analysis. Computational Statistics \& Data Analysis 109: 76-92.

5. Wei F, Tian W (2018) Heterogeneous connection effects. Statistics \& Probability Letters 133: 9-14.

\section{Your next submission with Juniper Publishers will reach you the below assets}

- Quality Editorial service

- Swift Peer Review

- Reprints availability

- E-prints Service

- Manuscript Podcast for convenient understanding

- Global attainment for your research

- Manuscript accessibility in different formats

( Pdf, E-pub, Full Text, Audio)

- Unceasing customer service

Track the below URL for one-step submission https://juniperpublishers.com/online-submission.php 